\title{
Miscibility of Main-Chain Thermotropic Polyesters
}

\author{
Jung-Il JIN, E-Joon CHOI, and Ki-Young LEE \\ Chemistry Department, College of Sciences, Korea University, \\ 1-Anam Dong, Seoul 132, Korea
}

(Received March 18, 1985)

\begin{abstract}
KEY WORDS Compatibility / Miscibility / Thermotropic Polyester / Nematic / Cholesteric / Phase Diagram / Optical Texture /
\end{abstract}

It is very well established theoretically as well as experimentally that polymers of different structures are in general incompatible with each other. ${ }^{1}$ Compatibility or incompatibility between polymers can be determined by various methods such as observation of changes in $T_{\mathrm{g}}$, optical clarity of a blend, construction of a phase diagram and miscibility of concentrated polymer solutions in a common solvent.

While pursuing an investigation of the relationship between the structure and properties of thermotropic main-chain polyesters, ${ }^{2-4}$ we found that the so-called miscibility rules of Demus and Sackmann ${ }^{5}$ appear to be applicable even to polymeric liquid crystals, although the number of systems to be reported by us here is very much limited. The rule was originally developed for the miscibility of low molar mass thermotropic compounds.

We believe that this is the first experimental study ever published on the miscibility of semiflexible liquid crystalline polymers showing phase diagrams covering whole range of composition of the mixtures. There are a few articles $^{6-8}$ which described only the miscibility of liquid crystalline polymers with low molar mass mesomorphic compounds.

Two different nematic polyesters, I and II and one cholesteric polyester were selected for the present study. Polymers I and II form nematic phase upon melting, while Polymer III cholesteric phase.

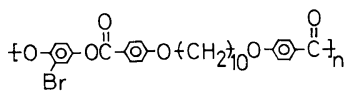

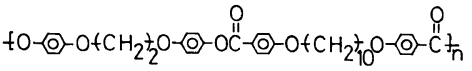

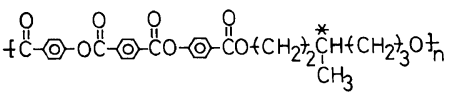

The preparation methods and properties of the polymers were reported earlier by $\mathrm{us}^{2,3,9}$ and others. ${ }^{10}$ Their mutual (I \& II and I \& III pairs) miscibility was studied by DSC (du Pont 910) and on a cross-polarizing microscope (Leitz, Ortholux) equipped with a hot-stage (Mettler FP-2):

Powderic polymers were first mixed thoroughly using a mortar and pestle. The mixture was transferred to a small test tube, which was then immersed in an oil bath and heated to isotropic state. The melts were continuously stirred with a glass rod. The hot test tube containing an isotropic mixture was allowed to stand at room temperature overnight in a desiccator. The mixtures were then utilized for DSC and microscopic studies. The heating rate for DSC analysis was $20 \mathrm{~K} \mathrm{~min}^{-1}$. The maximum positions of the heating endothermic peaks were taken as transition temperatures. DSC data agreed very well with the 
microscopic observations.

The phase diagrams obtained for the two systems are shown in Figures 1 and 2. Figure 3 shows DSC curves of Polymers I and II and a mixture thereof. Certainly the peak corresponding to isotropization of the mixture can be taken as a single endotherm comparing the shapes and widths of peaks from the homopolymers and the mixture. Figures 4 and 5 represent optical textures observed

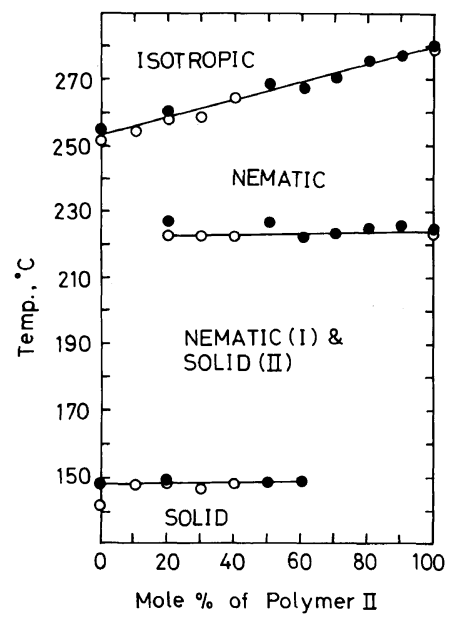

Figure 1. Phase diagram of the mixture of Polymer I and Polymer II. The $\mathrm{mol} \%$ of Polymer II shown in the diagram expresses the concentration of the repeat unit. The open circles $(O)$ are from the first DSC heating run and the filled ones (O) from the second heating. for the mixtures in the liquid crystalline states. Those results tell us that 1) the polymers did not form eutectic mixtures and the melting points of each polymers upon mixing were practically unaffected indicating their almost perfect immiscibility in the solid state, 2) nematic polymers are mutually miscible in the liquid crystalline phase and are isomorphous with cholesteric polymers, and 3) the transition temperature from a mesophase to an isotropic phase changed continuously and smoothly depending on the composition of the mixtures.

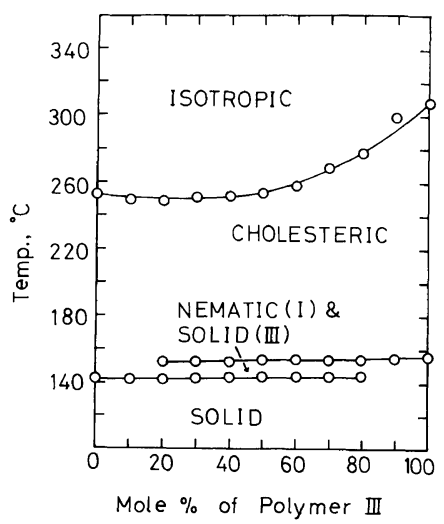

Figure 2. Phase diagram of the mixture of Polymer I and Polymer III. The $\mathrm{mol} \%$ of Polymer III shown in the diagram expresses the concentration of the repeat unit. All the data are from the first DSC heating.

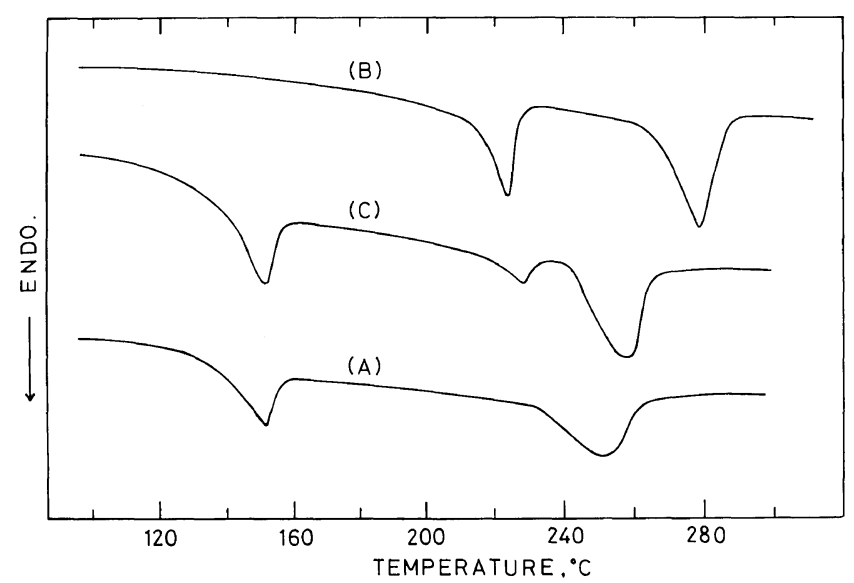

Figure 3. DSC curves of (a) Polymer I, (b) Polymer II and (c) a mixture of Polymers I and II in the repeating unit mole ratio of $8: 2$ obtained on the 2 nd heating. 


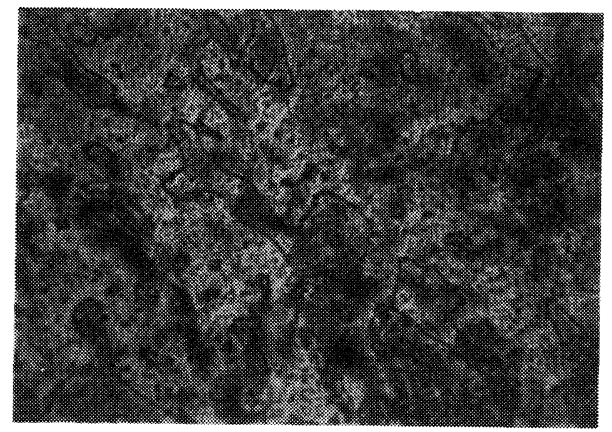

Figure 4. Microphotography for the equimolar mixture of Polymer I and Polymer II taken at $241^{\circ} \mathrm{C}$ (Magnification $100 \times$ ).

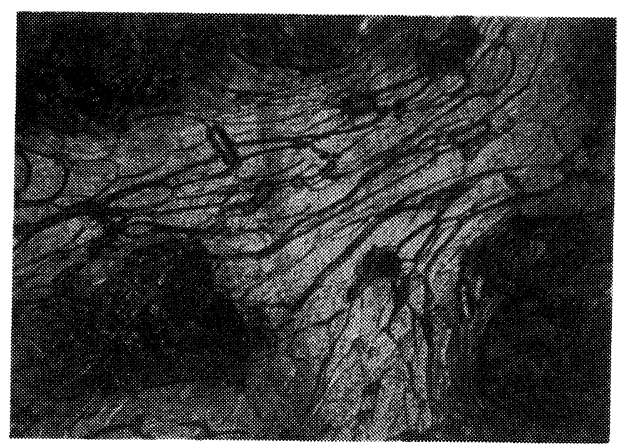

Figure 5. Microphotography for the equimolar mixture of Polymer I and Polymer III taken at $178^{\circ} \mathrm{C}$ (Magnification $100 \times$ ).

The optical textures of Figures 4 and 5 clearly demonstrate the homogeneous nature of the polymer mixtures in the mesophases. Prolonged maintenance of the melts in their mesophases on the hot-stage did not result in any phase separation when observed through a microscope. Figure 5 shows common oily streaks typical to cholesterics. On the contrary, a preliminary study on a mixture of a nematic and a smectic A polymers suggests that they are immiscible in the liquid crystalline state. ${ }^{11}$ Immiscibility between nematic and smectic polymers indicates that miscibility observed for the present two systems is not from the molecular blending caused by the mixing procedure.

The present findings offer us many important implications. First of all, miscibility of polymers of a same mesophase or being isomorphous can provide us with the new means of blending polymers which may lead to development of wide variety of materials properties that are impossible to obtain otherwise. Theoretical handling of such mixtures must also be very interesting and challenging. We are presently examining the miscibility of a wider variety of thermotropicpolymers.

Acknowledgment. The authors would like to express their thanks to the Korea Science and Engineering Foundation for the support of this work.

\section{REFERENCES}

1. O. Olabisi, L. M. Robeson, and M. T. Shaw, "Polymer-Polymer Miscibility," Academic Press, New York, 1979.

2. S. Antoun, J.-I. Jin, and R. W. Lenz, J. Polym. Sci., Polym. Chem. Ed., 19, 1901 (1981).

3. H.-J. Park, J.-I. Jin, and R. W. Lenz, Polymer, in press.

4. J.-I. Jin, S. Antoun, C. Ober, and R. W. Lenz, Br. Polym. J., 12, 132 (1980).

5. D. Demus and H. Sackmann, "Liquid Crystals," G. H. Brown, G. J. Dienes, and M. M. Labes, Ed., Gordon and Breach, New York, 1967, p 341.

6. A. C. Griffin and S. J. Havens, J. Polym. Sci., Polym. Lett. Ed., 18, 259 (1980).

7. E. R. George, R. S. Porter, and A. C. Griffin, Mol. Cryst. Liq. Cryst., 110, 27 (1984).

8. H. Finkelmann, H. J. Kock, and G. Rehage, Mol. Cryst. Liq. Cryst., 89, 23 (1982).

9. J.-I. Jin, B.-W. Jo, E-J. Choi, H.-T. Oh, and S.-C. Ryu, Presented at the Annual Meeting of the Polymer Society of Korea, October 19, 1984, ChunNam University, Kwang-Joo City, Korea.

10. A. C. Griffin and S. J. Havens, J. Polym. Sci., Polym. Phys. Ed., 19, 951 (1981).

11. J.-I. Jin, E-J. Choi, and K.-Y. Lee, Presented at the Annual Meeting of the Polymer Society of Korea, Kun-Kook University, Seoul, April 26, 1985. 